Mathematical Models and Methods in Applied Sciences

Vol. 26, No. 14 (2016) 2809-2814

(C) World Scientific Publishing Company

DOI: $10.1142 / \mathrm{S} 0218202516990013$

Abdulle, A. \& Pouchon, T., Effective models for the multidimensional wave equation in heterogeneous media over long time and numerical homogenization

Ahmedov, B., Grepl, M. A. \& Herty, M., Certified reduced-order methods for optimal treatment planning

Aihaiti, A., Enomoto, S. \& Kagei, Y., Large time behavior of solutions to the compressible NavierStokes equations in an infinite layer under slip boundary condition

Akrid, T., see Outada

Almeida, R. C., see Lima

Alonso, R., Amorim, P. \& Goudon, T., Analysis of a chemotaxis system modeling ant foraging

Alvarez, M., Gatica, G. N. \& Ruiz-Baier, R., A mixed-primal finite element approximation of a sedimentationconsolidation system

Ammari, H., Chen, D. \& Zou, J., Well-posedness of an electric interface model and its finite element approximation

Amorim, P., see Alonso

Andreianov, B., Donadello, C. \& Rosini, M. D., A second-order model for vehicular traffics with local point constraints on the flow

Banasiak, J., Falkiewicz, A. \& Namayanja, P., Asymptotic state lumping in transport and diffusion

\section{Author index Volume 26}

problems on networks with applications to population problems

26 (2016) 215

Bardos, C., Grebenkov, D. \& Rozanova-Pierrat, A., Short-time heat diffusion
26 (2016) 2651

26 (2016) 699

26 (2016) 2617

26 (2016) 2709

26 (2016) 2341

26 (2016) 1785

26 (2016) 867

26 (2016) 601

26 (2016) 1785

26 (2016) 751 in compact domains with discontinuous transmission boundary conditions

Barker, B. \& Zumbrun, K., Numerical proof of stability of viscous shock profiles

Barrett, J. W. \& Süli, E., Existence of global weak solutions to compressible isentropic finitely extensible bead-spring chain models for dilute polymers

Bayen, T., see Rapaport

Beirão da Veiga, L., Brezzi, F., Marini, L. D. \& Russo, A., Virtual Element Method for general second-order elliptic problems on polygonal meshes

26 (2016) 59

26 (2016) 2451

26 (2016) 469

26 (2016) 901

26 (2016) 729

Beirão da Veiga, L., Chernov, A., Mascotto, L. \& Russo, A., Basic principles of $h p$ virtual elements on quasiuniform meshes

Bellomo, N. \& Brezzi, F., Mathematics, complexity and multiscale features of large systems of selfpropelled particles

26 (2016) 207

Bellomo, N., Bellouquid, A. \& Chouhad, N., From a multiscale derivation of nonlinear cross-diffusion models to Keller-Segel models in a Navier-Stokes fluid

26 (2016) 2041

Bellomo, N., see Marsan

Bellouquid, A., Nieto, J. \& 26 (2016) 1051 
kinetic description of fractional diffusion equations modeling chemotaxis

Bellouquid, A., see Bellomo

Beretta, E., Cristina Cerutti, M., Manzoni, A. \& Pierotti, D., An asymptotic formula for boundary potential perturbations in a semilinear elliptic equation related to cardiac electrophysiology

Borsche, R., Kall, J., Klar, A. \& Pham, T. N. H., Kinetic and related macroscopic models for chemotaxis on networks

Brezzi, F., see Bellomo

Brezzi, F., see Beirão da Veiga

Buffa, A. \& Giannelli, C., Adaptive isogeometric methods with hierarchical splines: Error estimator and convergence

Bulíček, M., Gwiazda, P., Süli, E. \& ŚwierczewskaGwiazda, A., Analysis of a viscosity model for concentrated polymers

Burczak, J. \& GraneroBelinchón, R., On a generalized doubly parabolic Keller-Segel system in one spatial dimension

Carrillo, J. A., Choi, Y.-P., Tadmor, E. \& Tan, C., Critical thresholds in 1D Euler equations with nonlocal forces

Carrillo, J. A., Martin, S. \& Wolfram, M.-T., An improved version of the Hughes model for pedestrian flow

Carrillo, J. A., Choi, Y.P. \& Zatorska, E., On the pressureless damped Euler-Poisson equations with quadratic confinement: Critical thresholds and large-time behavior

Chen, D., see Ammari

Chen, S., see Zhao

Chernov, A., see Beirão da Veiga

Cho, J., Ha, S.-Y., Huang, F., Jin, C. \& Ko, D., Emergence of bi-cluster flocking for the CuckerSmale model
26 (2016) 249

26 (2016) 2041

26 (2016) 645

26 (2016) 1219

26 (2016) 207

26 (2016) 729

26 (2016) 1

26 (2016) 1599

26 (2016) 111

26 (2016) 185

26 (2016) 671

26 (2016) 2311

26 (2016) 601

26 (2016) 1671

26 (2016) 1567

26 (2016) 1191
Choi, Y.-P., see Carrillo

Choi, Y.-P., see Carrillo

Chouhad, N., see Bellomo

Cicalese, M., Ruf, M. \&

Solombrino, F., Chirality transitions in frustrated $S^{2}$-valued spin systems

Colli Franzone, P., Pavarino, L. F. \& Scacchi, S., Bioelectrical effects of mechanical feedbacks in a strongly coupled cardiac electro-mechanical model

Colombo, M. \& Stra, F., Counterexamples in multimarginal optimal transport with Coulomb cost and spherically symmetric data

Colombo, R. M. \& Marcellini, F., A traffic model aware of real time data

Conti, S. \& Zwicknagl, B., Low volumefraction microstructures in martensites and crystal plasticity

Cristina Cerutti, M., see Beretta

Cucker, F. \& Dong, J.-G., On flocks influenced by closest neighbors

Davoli, E., Piovano, P. \& Stefanelli, U., Wulff shape emergence in graphene

Degond, P., Delebecque, F. \& Peurichard, D., Continuum model for linked fibers with alignment interactions

Delebecque, F., see Degond

Dellacherie, S., Jung, J., Omnes, P. \& Raviart, P.-A., Construction of modified Godunov-type schemes accurate at any Mach number for the compressible Euler system

Di Francesco, M. \& Fagioli, S., A nonlocal swarm model for predators-prey interactions

Di Marino, S. \& Mészáros, A. R., Uniqueness issues for evolution equations with density constraints

Diaz, J. \& Péron, V., Equivalent Robin boundary conditions for acoustic and elastic media
26 (2016) 185

26 (2016) 2311

26 (2016) 2041

26 (2016) 1481

26 (2016) 27

26 (2016) 1025

26 (2016) 445

26 (2016) 1319

26 (2016) 645

26 (2016) 2685

26 (2016) 2277

26 (2016) 269

26 (2016) 269

26 (2016) 2525

26 (2016) 319

26 (2016) 1761

26 (2016) 1531 
Dimarco, G. \& Motsch, S., Self-alignment driven by jump processes: Macroscopic limit and numerical investigation

Donadello, C., see Andreianov

Dong, J.-G., see Cucker

Donoso-Bravo, A., see Rapaport

Enomoto, S., see Aihaiti

Fagioli, S., see Di Francesco

Falkiewicz, A., see Banasiak

Fang, J. \& Faye, G., Monotone traveling waves for delayed neural field equations

Faye, G., see Fang

Feireisl, E., Klein, R., Novotný, A. \& Zatorska, E., On singular limits arising in the scale analysis of stratified fluid flows

Frigeri, S., Global existence of weak solutions for a nonlocal model for two-phase flows of incompressible fluids with unmatched densities

Garcke, H., Lam, K. F., Sitka, E. \& Styles, V., A Cahn-Hilliard-Darcy model for tumour growth with chemotaxis and active transport

Garcke, H., Kampmann, J., Rätz, A. \& Röger, M., A coupled surface-CahnHilliard bulk-diffusion system modeling lipid raft formation in cell membranes

Garroni, A., van Meurs, P., Peletier, M. A. \& Scardia, L., Boundary-layer analysis of a pile-up of walls of edge dislocations at a lock

Gatica, G. N., see Alvarez

Giannelli, C., see Buffa

Gibelli, L., see Marsan

Goudon, T., see Alonso

Granero-Belinchón, R., see Burczak

Grebenkov, D., see Bardos

Grepl, M. A., see Ahmedov

Grün, G. \& Metzger, S., On micro-macro-models for two-phase flow with dilute polymeric solutions Modeling and analysis
Gwiazda, P., see Bulíček

Ha, S.-Y., see Li

Ha, S.-Y., see Cho

Herty, M., see Ahmedov

26 (2016) 1385

26 (2016) 751

26 (2016) 2685

26 (2016) 901

26 (2016) 2617

26 (2016) 319

26 (2016) 215

26 (2016) 1919

26 (2016) 1919

26 (2016) 419

26 (2016) 1955

26 (2016) 1095

26 (2016) 1149

26 (2016) 2735

26 (2016) 867

26 (2016) 1

26 (2016) 1051

26 (2016) 1785

26 (2016) 111

26 (2016) 59

26 (2016) 699

26 (2016) 823 production lower order case

Huang, F., see Cho Boltzmann system Jin, C., see Cho symmetry

Jung, J., see Dellacherie

Kagei, Y., see Aihaiti

Kall, J., see Borsche problems

Khaladi, M., see Outada tion

Klar, A., see Borsche

Klein, R., see Feireisl

Ko, D., see Cho

Kwon, B., see Jung

Lam, K. F., see Garcke
26 (2016) 1599

26 (2016) 357

26 (2016) 1191

26 (2016) 699

26 (2016) 161

26 (2016) 2341

26 (2016) 2111

Hu, J. \& Zhang, S., Finite element approximations of symmetric tensors on simplicial grids in $\mathbb{R}^{n}$ : The

Huang, L. \& Liu, S., Stability of contact discontinuity for the twospecies Vlasov-Poisson-

Hughes, T. J. R., see Taus

Jung, C.-Y., Kwon, B. \& Suzuki, M., Quasineutral limit for the Euler-Poisson system in the presence of plasma sheaths with spherical

Kampmann, J., see Garcke

Kateb, D. \& Le Louër, F., Generalized impedance boundary conditions and shape derivatives for 3D Helmholtz

Kitavtsev, G., Robbins, J. M., Slastikov, V. \& Zarnescu, A., Liquid crystal defects in the Landaude Gennes theory in two dimensions - Beyond the one-constant approxima-
26 (2016) 1649 26 (2016) 1191

26 (2016) 1243 26 (2016) 1191

26 (2016) 2369

26 (2016) 2525

26 (2016) 2617

26 (2016) 1219

26 (2016) 1149

26 (2016) 1995

26 (2016) 2709

26 (2016) 2769

26 (2016) 1219

26 (2016) 419

26 (2016) 1191

26 (2016) 2369

26 (2016) 1095
26 (2016) 1447 
Lankeit, J., Longterm behaviour in a chemotaxis-fluid system with logistic source

Lattanzio, C., Mascia, C., Plaza, R. G. \& Simeoni, C., Analytical and numerical investigation of traveling waves for the AllenCahn model with relaxation

Le Louër, F., see Kateb

Leonardi, F., Mishra, S. \& Schwab, C., Numerical approximation of statistical solutions of planar, incompressible flows

Li, J., Titi, E. S. \& Xin, Z., On the uniqueness of weak solutions to the EricksenLeslie liquid crystal model in $\mathbb{R}^{2}$

Li, Z. \& Ha, S.-Y., Uniqueness and well-ordering of emergent phase-locked states for the Kuramoto model with frustration and inertia

Liljegren-Sailer, B., see Marheineke

Lima, E. A. B. F., Oden, J. T., Hormuth, II, D. A., Yankeelov, T. E. \& Almeida, R. C., Selection, calibration, and validation of models of tumor growth

Liu, S., see Huang

Lorenz, M., see Marheineke

Mácha, V. \& Nečasová, Š., Self-propelled motion in a viscous compressible fluid — Unbounded domains

Maggiani, G. B. \& Mora, M. G., A dynamic evolution model for perfectly plastic plates

Manzoni, A., see Beretta

Marcellini, F., see Colombo

Marheineke, N., LiljegrenSailer, B., Lorenz, M. \& Wegener, R., Asymptotics and numerics for the upper-convected Maxwell model describing transient curved viscoelastic jets

Marini, L. D., see Beirão da Veiga
Marsan, G. A., Bellomo, N. \& Gibelli, L., Stochastic evolutionary differential games toward a systems theory of behavioral social dynamics

Martin, S., see Carrillo

Mascia, C., see Lattanzio

Mascotto, L., see Beirão da Veiga

26 (2016) 931

26 (2016) 1995

Mészáros, A. R., see Di Marino

Metzger, S., see Grün

Mielke, A. \& Roubíček, T. Rate-independent elastoplasticity at finite strains and its numerical approximation

26 (2016) 2471

Mishra, S., see Leonardi

Mora, M. G., see Maggiani

Motsch, S., see Dimarco

26 (2016) 803

Namayanja, P., see Banasiak

Nečasová, S̆., see Mácha

Nieto, J., see Bellouquid

Nochetto, R. H., Salgado, A. J. \& Tomas, I., The equations of ferrohydrodynam-

26 (2016) 357

26 (2016) 569 ical methods

Novotný, A., see Feireisl

Oden, J. T., see Lima

Omnes, P., see Dellacherie

Outada, N., Vauchelet, N., Akrid, T. \& Khaladi, M., From kinetic theory of multicellular systems to hyperbolic tissue equations: Asymptotic limits and computing

Pavarino, L. F., see Colli Franzone

Peletier, M. A., see Garroni

Péron, V., see Diaz

26 (2016) 627

Perversi, E. \& Regazzini, E., Inequality and risk aversion in economies open to altruistic attitudes

26 (2016) 1825

26 (2016) 645

26 (2016) 445

Peurichard, D., see Degond

Pham, T. N. H., see Borsche

Piccoli, B., Pouradier Duteil, N. \& Scharf, B., Optimal control of a collective migration model

Pierotti, D., see Beretta

Piovano, P., see Davoli

Plaza, R. G., see Lattanzio

Popoff, N., see Hislop

26 (2016) 569

Pouchon, T., see Abdulle

Pouradier Duteil, N., see Piccoli
26 (2016) 1051

26 (2016) 671

26 (2016) 931

26 (2016) 1567

26 (2016) 1761

26 (2016) 823

26 (2016) 2203

26 (2016) 2471

26 (2016) 1825

26 (2016) 1385

26 (2016) 215

26 (2016) 627

26 (2016) 249

26 (2016) 2393

26 (2016) 419

26 (2016) 2341

26 (2016) 2525

26 (2016) 2709

26 (2016) 27

26 (2016) 2735

26 (2016) 1531

26 (2016) 1735

26 (2016) 269

26 (2016) 1219

26 (2016) 383

26 (2016) 645

26 (2016) 2277

26 (2016) 931

26 (2016) 161

26 (2016) 2651

26 (2016) 383 
Rapaport, A., Bayen, T., Sebbah, M., DonosoBravo, A. \& Torrico, A., Dynamical modeling and optimal control of landfills

Rätz, A., see Garcke

Raviart, P.-A., see Dellacherie

Raymond, N., see Hislop

Regazzini, E., see Perversi

Robbins, J. M., see Kitavtsev

Rodin, G. J., see Taus

Röger, M., see Garcke

Rosini, M. D., see Andreianov

Roubíček, T., see Mielke

Rozanova-Pierrat, A., see Bardos

Ruf, M., see Cicalese

Ruiz-Baier, R., see Alvarez

Russo, A., see Beirão da Veiga

Russo, A., see Beirão da Veiga

Salgado, A. J., see Nochetto

Savostianov, A. \& Zelik, S., Global well-posedness and attractors for the hyperbolic Cahn-Hilliard-Oono equation in the whole space

Scacchi, S., see Colli Franzone

Scardia, L., see Garroni

Scharf, B., see Piccoli

Schwab, C., see Leonardi

Sebbah, M., see Rapaport

Segatti, A., Snarski, M. \& Veneroni, M., Analysis of a variational model for nematic shells

Simeoni, C., see Lattanzio

Sitka, E., see Garcke

Slastikov, V., see Kitavtsev

Snarski, M., see Segatti

Solombrino, F., see Cicalese

Stefanelli, U., see Davoli

Stinner, C., Surulescu, C. \& Uatay, A., Global existence for a go-orgrow multiscale model for tumor invasion with therapy

Stra, F., see Colombo

Styles, V., see Garcke

Süli, E., see Barrett

Süli, E., see Bulíček

Sundqvist, M. P., see Hislop

Surulescu, C., see Stinner
26 (2016) 2525

26 (2016) 161

26 (2016) 1735

26 (2016) 2769

26 (2016) 1447

26 (2016) 1149

26 (2016) 751

26 (2016) 2203

26 (2016) 59

26 (2016) 1481

26 (2016) 867

26 (2016) 729

26 (2016) 1567

26 (2016) 2393

26 (2016) 1357

26 (2016) 27

26 (2016) 2735

26 (2016) 383

26 (2016) 2471

26 (2016) 901

26 (2016) 1865

26 (2016) 931

26 (2016) 1095

26 (2016) 2769

26 (2016) 1865

26 (2016) 1481

26 (2016) 2277

26 (2016) 2163

26 (2016) 1025

26 (2016) 1095

26 (2016) 469

26 (2016) 1599

26 (2016) 161

26 (2016) 2163
Suzuki, M., see Jung

Świerczewska-Gwiazda, A., see Bulíček

Tadmor, E., see Carrillo

Takacs, S. \& Takacs, T., Approximation error estimates and inverse inequalities for B-splines of maximum smoothness

26 (2016) 2369

26 (2016) 1599

26 (2016) 185

26 (2016) 1411

26 (2016) 1411

26 (2016) 185

26 (2016) 2111

Tao, Y., see $\mathrm{Hu}$

Taus, M., Rodin, G. J. \& Hughes, T. J. R., Isogeometric analysis of boundary integral equations: High-order collocation methods for the singular and hyper-singular equations

Tello, J. I. \& Wrzosek, D., Predator-prey model with diffusion and indirect prey-taxis

Titi, E. S., see Li

Tomas, I., see Nochetto

Torrico, A., see Rapaport

Uatay, A., see Stinner

Urrutia, L., see Bellouquid

van Meurs, P., see Garroni

Vauchelet, N., see Outada

Veneroni, M., see Segatti

Wang, T. \& Zhao, H., Onedimensional compressible heat-conducting gas with temperature-dependent viscosity

Wegener, R., see Marheineke

Winkler, M., The twodimensional Keller-Segel system with singular sensitivity and signal absorption: Global large-data solutions and their relaxation properties

Winkler, M., Chemotactic cross-diffusion in complex frameworks

Wolfram, M.-T., see Carrillo

Wrzosek, D., see Tello

Xiao, J., Global weak entropy solutions to the Euler-Poisson system with spherical symmetry

Xin, Z., see $\mathrm{Li}$

Yankeelov, T. E., see Lima

Zarnescu, A., see Kitavtsev

Zatorska, E., see Feireisl

Zatorska, E., see Carrillo
26 (2016) 1447

26 (2016) 2129

26 (2016) 803

26 (2016) 2393

26 (2016) 901

26 (2016) 2163

26 (2016) 249

26 (2016) 2735

26 (2016) 2709

26 (2016) 1865

26 (2016) 2237

26 (2016) 569

26 (2016) 987

26 (2016) 2035

26 (2016) 671

26 (2016) 2129

26 (2016) 1689

26 (2016) 803

26 (2016) 2341

26 (2016) 2769

26 (2016) 419

26 (2016) 2311 
Zelik, S., see Savostianov

Zhang, B., see Zhao

Zhang, S., see $\mathrm{Hu}$

Zhao, H., see Wang

Zhao, J., Chen, S. \& Zhang,

B., The nonconforming
26 (2016) 1357

26 (2016) 1671

26 (2016) 1649

26 (2016) 2237 virtual element method for plate bending problems

Zou, J., see Ammari

Zumbrun, K., see Barker

Zwicknagl, B., see Conti
26 (2016) 1671

26 (2016) 601

26 (2016) 2451

26 (2016) 1319 